\title{
Phase phonon spectrum and melting in a quantum rotor model with diagonal disorder
}

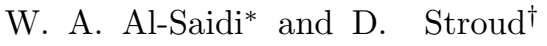 \\ Department of Physics, The Ohio State University, Columbus, Ohio 43210
}

(Dated: November 13, 2018)

\begin{abstract}
We study the zero-temperature $(T=0)$ quantum rotor model with on-site disorder in the charging energy. Such a model may serve as an idealized Hamiltonian for an array of Josephson-coupled small superconducting grains, or superfluid ${ }^{4} \mathrm{He}$ in a disordered environment. In the approximation of small-amplitude phase fluctuations, the Hamiltonian maps onto a system of coupled harmonic oscillators with on-site disorder. We study the effects of disorder in this harmonic regime, using the coherent potential approximation (CPA), obtaining the density of states and the lifetimes of the spin-wave-like excitations for several choices of the parameters which characterize the disorder. Finally, we estimate the parameters characterizing the $T=0$ quantum melting of the phase order, using a suitable Lindemann criterion.

PACS numbers: 03.65.Ud, 03.67.Lx, 42.50.Fx, 74.50.+r
\end{abstract}

\section{INTRODUCTION}

The quantum rotor model is a widely studied Hamiltonian which may serve as an idealized representation of many physical systems. It consists of two parts: a potential energy which represents the coupling between two-component spin variables of fixed length (an "XY" Hamiltonian) and the kinetic energy of those spins. The model is quantum-mechanical because the potential and kinetic energies do not commute.

One possible realization of this model is a Josephson junction array, i.e., a collection of superconducting islands with Josephson or proximity effect tunneling between the islands. Such arrays can be produced experimentally in a wide range of geometries, and with a great variety of individual junction parameters. They may be described by the quantum rotor model in the limit of small grains. In this case the potential energy corresponds to the Josephson coupling, and the kinetic energy corresponds to the charging energy. Such arrays can serve as model systems for studying quantum phase transitions 1 under conditions such that the experimental parameters can be readily tuned.6 Recently, it has also been proposed that Josephson junctions may serve as a quantum bit (qubit) in quantum information technology, where the quantum logic operations would be performed by controlling gate voltages or magnetic fields. 3

The quantum rotor model may also be realized in other physical systems. For example, in liquid ${ }^{4} \mathrm{He}$, the potential energy may represent a discretized form of the gradient energy term in the Ginzburg-Landau free energy of superfluid ${ }^{4} \mathrm{He}$, and favors a state in which all parts of the superfluid have the same phase, while the kinetic energy term is the energy associated with the time-variation of the order parameter phase. The model may be particularly useful for helium films and helium in confined geometries such as porous glasses. The spectrum of elementary excitations in such confined geometries has recently been studied extensively. 4 This spectrum may be treatable by the quantum rotor model.

In this paper, we study a special kind of quantum ro- tor model, namely, the two-dimensional model with diagonal disorder in the kinetic energy. Our emphasis in this study is to understand what happens to the spectrum of elementary excitations in the presence of this disorder. We also investigate the conditions for the destruction of phase order in this model arising from such quantum fluctuations in the phase variables. This model includes a particularly simple form of disorder. In the case of a Josephson junction array (JJA), it would correspond to fluctuations in the self-capacitance of individual grains, but no significant randomness in the Josephson coupling between the superconducting grains. In the case of superfluid helium, the diagonal disorder might correspond to randomness in the pore geometry (for a three-dimensional system) or arising from surface roughness (for a thin film).

In the present paper, we are primarily interested in the spectrum of elementary excitations in this model at low temperatures. We therefore adopt a simple meanfield approximation which has been successfully used to treat elementary excitations in other systems. This is the coherent potential approximation (CPA), as described originally by Soven, $\mathrm{D}$ Taylor et al, , $\mathrm{E}_{\mathrm{H}}$ and Velický et al. For the present problem, the CPA may be adequate at very low temperatures, under conditions such that the original Hamiltonian can be approximated as harmonic. At higher temperatures, or under conditions such that the harmonic approximation is inadequate, it will be necessary to consider a more sophisticated approximation, such as the self-consistent harmonic approximation (SCHA), 10.11 or a field-theoretic treatment in which the charging energy is mapped onto a coupling in the imaginary time direction. 12

The remainder of this paper is organized as follows. In the next section, we present the model Hamiltonian, and its relation to a set of coupled harmonic oscillators. In Sec. III, we describe how the CPA may be used to study the disorder-averaged properties of the model Hamiltonian in the harmonic approximation. Our numerical results based on the CPA are presented in Sec. IV, followed by a concluding discussion in Sec. V. 


\section{MODEL HAMILTONIAN}

We will consider the following model Hamiltonian:

$$
H=\frac{1}{2} \sum_{j} U_{j} n_{j}^{2}+\sum_{j k} J_{j k}\left[1-\cos \left(\theta_{j}-\theta_{k}\right)\right] .
$$

Here $\theta_{j}$ is the phase of the quantum rotor, and $n_{j}$ is a variable which is quantum-mechanically conjugate to the angle $\theta_{j} . \quad n_{j}$ and $\theta_{k}$ are assumed to have commutation relations

$$
\left[n_{j}, \theta_{k}\right]=-i \delta_{j k}
$$

where $\delta_{j k}$ is a Kronecker delta function and [...] represents a commutator. In Eq. (11), the first sum runs over sites $j$, and the second sum runs over all distinct pairs of sites $j$ and $k$. If this model is assumed to describe a Josephson junction array, then the first term represents the capacitive energy of the array in the diagonal approximation. The most general form of this capacitive energy would be $\frac{1}{2} \sum_{j k} U_{j k} n_{j} n_{k}$; in Eq. (1), we make the approximation $U_{j k}=U_{j} \delta_{j k}$, where $U_{j}$ is related to the diagonal capacitance $C_{j}$, or alternatively to an effective "mass" $M_{j}$,

$$
U_{j}=\frac{4 e^{2}}{C_{j}} \equiv \frac{\hbar^{2}}{M_{j}}
$$

The second term represents the sum of the Josephson coupling energies between grains $j$ and $k$. In Eq. (11), it is implicitly assumed that there is no contribution to $H$ arising from dissipation. In this paper, we shall assume that $J_{i j} \neq 0$ only if $i$ and $j$ are nearest-neighbor grains, and that, furthermore, there is no disorder in this nearest-neighbor coupling, i.e., that $J_{i j}=J$ for all nearest neighbor pairs $i$ and $j$. Hereafter, we shall refer to this model Hamiltonian as describing a Josephson junction array, although, as noted above, the model is also applicable, in principle, to other physical systems.

For sufficiently low temperatures, we assume that the above Hamiltonian can be approximated by its harmonic form:

$$
H_{\mathrm{harm}}=\frac{1}{2} \sum_{j} U_{j} n_{j}{ }^{2}+\frac{1}{2} J \sum_{\langle j k\rangle}\left(\theta_{j}-\theta_{k}\right)^{2} \equiv K+V,
$$

where the second sum runs over distinct nearest neighbor pairs. While one can go beyond the harmonic approximation by using a self-consistent phase phonon approach, 10 we shall here consider only the harmonic approximation, which is more easily combined with a treatment of disorder. The Heisenberg equation of motion of the phase variable is $i \hbar \dot{\theta}_{j}=\left[\theta_{j}, H\right]=i U_{j} n_{j}$, where the last equality follows from the commutation relation (2). This equation makes clear that $H_{\text {harm }}$ is formally analogous to an array of "masses" $M_{j}$ harmonically coupled by springs of "spring constant" J. However, in contrast to a real lattice of masses and springs, there is no polarization degree of freedom. $K$ and $V$ represent the kinetic and potential energy terms in this Hamiltonian. A disordered array of such oscillators can thus be treated by the same methods used to treat disordered arrays of coupled oscillators.

In this paper, we shall consider a bimodal distribution of the $U_{j}$ 's. Thus we take $U_{j}=U_{1}$ or $U_{2}$ with probabilities $p$ and $1-p$ respectively. We also assume that the type 1 and type 2 grains are randomly distributed on an ideal lattice, with no correlation between the occupancies of neighboring sites. With this choice, the Hamiltonian (4) is analogous to the classic problem of substitutional mass disorder in phonon spectra, except that the "phase phonon" excitations have only one possible polarization. This problem has previouslybeen studied by Soven, 5 Taylor 6 , and Elliott and Taylor, 0 using the coherent potential approximation. 6.0 In the present paper, we shall use the same approach to study the effects of disorder in the "phase phonon" spectrum. Note that because the entire Hamiltonian is invariant under a global rotation of phases, the long-wavelength phase phonons are Goldstone bosons with frequencies that go to zero linearly in the wave vector $\mathbf{k}$, even in the presence of disorder, and even in two dimensions.

\section{COHERENT POTENTIAL APPROXIMATION}

\section{A. General equations}

The CPA provides an approximate way of calculating the disorder-averaged Green's function

$$
\bar{G}(z) \equiv\left\langle\frac{1}{z-H_{\text {harm }}}\right\rangle_{\text {dis }},
$$

where $z$ is a complex variable, $H_{\text {harm }}$ is the Hamiltonian operator, and $\langle\ldots\rangle_{\text {dis }}$ represent an average over disorder realizations. Of particular interest is the diagonal matrix element of this operator, which we write

$$
F(z)=\langle j|\bar{G}(z)| j\rangle \equiv \bar{G}(j, j ; z) .
$$

Because the operator $\bar{G}$ is disorder-averaged, $F(z)$ is independent of $j$.

Also, since $\bar{G}(z)$ is disorder-averaged, it can be expressed in terms of a periodic operator $H_{\text {eff }}$ by the relation

$$
\bar{G}(z)=\frac{1}{z-H_{\mathrm{eff}}} .
$$

For the present case of "site-diagonal" disorder, $H_{\mathrm{eff}}$ can be written

$$
H_{\mathrm{eff}}=K_{\mathrm{eff}}+V
$$

where $K_{\text {eff }}$ can be expressed as

$$
K_{\mathrm{eff}}=\frac{\hbar^{2}}{2 M_{\mathrm{eff}}} \sum_{j} n_{j}^{2}
$$


Here $M_{\text {eff }}(z)$ is a complex, frequency-dependent "effective mass" which is, however, the same for each site $j$. It is convenient to express $M_{\text {eff }}$ in terms of two other quantities - the "virtual-crystal" mass

$$
M_{\mathrm{VCA}}=p M_{1}+(1-p) M_{2},
$$

and a dimensionless, complex self-energy function $\Sigma(z)$ defined by

$$
M_{\mathrm{eff}}(z)=M_{\mathrm{VCA}}[1-\Sigma(z)] .
$$

In the CPA, it is found that $F\left(z^{2}\right)$ and $\Sigma(z)$ are connected by the complex scalar equation 13

$$
M_{\mathrm{VCA}} \Sigma(z)=\frac{p(1-p)(\delta M)^{2} z^{2} F\left(z^{2}\right)}{1+z^{2}\left[(1-2 p) \delta M+M_{\mathrm{VCA}} \Sigma(z)\right] F\left(z^{2}\right)} .
$$

Here $\delta M \equiv M_{1}-M_{2} \equiv-\epsilon M_{2}$, where $\epsilon \equiv 1-M_{1} / M_{2}=$ $1-C_{1} / C_{2}$, and we have adopted the convention that $C_{1}<C_{2}$.

In order to complete the approximation, we need to express $F\left(z^{2}\right)$ in terms of $\Sigma(z)$. This is readily accomplished as follows. First, we consider the analogous virtual-crystal Green's function $G_{\mathrm{VCA}}(z) \equiv 1 /(z-$ $\left.K_{\mathrm{VCA}}-V\right)$, where $K_{\mathrm{VCA}}=\sum_{j} n_{j}^{2} /\left[2 M_{\mathrm{VCA}}\right]$. Since $K_{\mathrm{VCA}}+V$ is periodic, $G_{\mathrm{VCA}}\left(z^{2}\right)$ is diagonal in momentum space. In particular, the diagonal matrix element is given by

$$
\left\langle\mathbf{q}\left|G_{\mathrm{VCA}}\left(z^{2}\right)\right| \mathbf{q}\right\rangle=\frac{1}{z^{2}-\omega_{\mathbf{q}}^{2}} \equiv G_{\mathrm{VCA}}\left(\mathbf{q} ; z^{2}\right),
$$

where $\omega_{\mathbf{q}}$ is the frequency of a "phase phonon" of wave vector $\mathbf{q}$ in the virtual crystal, and $|\mathbf{q}\rangle$ and $|i\rangle$ are related by $|\mathbf{q}\rangle=N^{-1 / 2} \sum_{i} e^{i \mathbf{q} \cdot \mathbf{R}_{i}}|i\rangle, \mathbf{R}_{i}$ being the position of the $i$ th grain and $N$ being the number of grains in the lattice (which may be assumed to have periodic boundary conditions). The corresponding diagonal matrix element $F_{\mathrm{VCA}}\left(z^{2}\right)$ is given by

$$
F_{\mathrm{VCA}}\left(z^{2}\right)=\frac{1}{2 N M_{\mathrm{VCA}}} \sum_{\mathbf{q}} G_{\mathrm{VCA}}\left(\mathbf{q}, z^{2}\right),
$$

where the sum runs over q's in the first Brillouin zone of the grain lattice. Next, we introduce the virtual-crystal density of states $g_{\mathrm{VCA}}(\eta)$ by

$$
g_{\mathrm{VCA}}(\eta)=\frac{1}{2 N} \sum_{\mathbf{q}} \delta\left(\eta-\omega_{\mathbf{q}}\right),
$$

in terms of which $F_{\mathrm{VCA}}\left(z^{2}\right)$ takes the form

$$
F_{\mathrm{VCA}}\left(z^{2}\right)=\frac{1}{M_{\mathrm{VCA}}} \int_{0}^{\infty} \frac{g_{\mathrm{VCA}}(\eta)}{z^{2}-\eta^{2}} d \eta
$$

Finally, we can obtain $F\left(z^{2}\right)$ for the actual disordered crystal in terms of $F_{\mathrm{VCA}}\left(z^{2}\right)$ simply by making the replacement $z^{2} \rightarrow z^{2}[1-\Sigma(z)]$, so that

$$
\begin{aligned}
F\left(z^{2}\right) & =F_{\mathrm{VCA}}\left(z^{2}[1-\Sigma(z)]\right) \\
& =\frac{1}{M_{\mathrm{VCA}}} \int_{0}^{\infty} \frac{g_{\mathrm{VCA}}(\eta)}{z^{2}[1-\Sigma(z)]-\eta^{2}} d \eta .
\end{aligned}
$$

Equations. (12) and (17) represent two coupled equations for $F\left(z^{2}\right)$ and $\Sigma(z)$ which can be solved simultaneously, given a model for $g_{\mathrm{VCA}}(\eta)$.

In the present paper, we will consider a twodimensional (2D) lattice with a simple Debye-like density of states defined by

$$
g_{\mathrm{VCA}}(\eta)=\frac{\eta}{2 \pi v^{2}} \Theta\left(\omega_{D}-\eta\right) .
$$

Here $v=\sqrt{J / M_{\mathrm{VCA}}}$ is the speed of the "phase phonon" excitations in the virtual crystal, and $\Theta\left(\omega_{D}-\eta\right)$ is a unit step function. The Debye cutoff frequency

$$
\omega_{D}=\left(4 \pi v^{2}\right)^{1 / 2}=\left(4 \pi J / M_{\mathrm{VCA}}\right)^{1 / 2},
$$

follows from requiring that the number of phase phonon states be normalized to one per grain. The quantity $F_{\mathrm{VCA}}\left(z^{2}\right)$ follows readily from Eq. (16):

$$
F_{\mathrm{VCA}}\left(z^{2}\right)=\frac{1}{M_{\mathrm{VCA}} \omega_{D}^{2}} \ln \left[\frac{z^{2}}{z^{2}-\omega_{D}^{2}}\right] .
$$

Equations (12), (17), and (20) constitute a self-consistent set of equations which can now be explicitly solved to give the properties of the phase excitations in the disordered system, within the CPA.

\section{B. Lattice properties}

\section{Density of states}

The density of phase phonon excitations within the $\mathrm{CPA}$ is given by 13

$$
g_{\mathrm{CPA}}(\omega)=-\frac{2}{\pi \omega} \operatorname{Im} \int_{0}^{\infty} \frac{\eta^{2} g_{\mathrm{VCA}}(\eta)}{\omega^{2}[1-\Sigma(\omega)]-\eta^{2}} d \eta .
$$

Using Eq. (18) for $g_{\mathrm{VCA}}(\eta)$, this expression can be further simplified to

$$
g_{\mathrm{CPA}}(\omega)=-\frac{2}{\pi \omega} \operatorname{Im}\left[h\left(x^{2}\right)\right],
$$

where $x^{2}=\left(\omega^{2} / \omega_{D}^{2}\right)[1-\Sigma(\omega)]$ and $h\left(x^{2}\right)=$ $x^{2} \ln \left[x^{2} /\left(x^{2}-1\right)\right]-1$. Thus, once we have obtained the self-energy from Eq. (12), we can find the density of vibrational states, $g_{\mathrm{CPA}}(\omega)$ from Eq. (22). 14

The CPA can also be used to give the component densities of states $g_{\mathrm{CPA}}^{(\alpha)}(\omega)(\alpha=1,2)$, defined to be the average density of phase phonon density of states on grains of type $\alpha$. This is obtained in terms of the average diagonal matrix element $\bar{G}^{(\alpha)}\left(j, j ; z^{2}\right)$ for a grain of type $\alpha$. In the $\mathrm{CPA}$, this matrix element takes the form:

$$
\bar{G}^{\alpha}\left(j, j ; z^{2}\right)=\frac{F\left(z^{2}\right)}{1-\left(M_{\mathrm{VCA}}-M_{\alpha}-M_{\mathrm{VCA}} \Sigma\right) z^{2} F\left(z^{2}\right)},
$$

where $F\left(z^{2}\right)$ is defined in Eq. (6). The average density of states at a grain of type $\alpha$ (normalized so as to integrate to unity over positive frequencies) is given by 


$$
g_{\mathrm{CPA}}^{(\alpha)}(\omega)=-\frac{2}{\pi \omega} \operatorname{Im}\left[\frac{h\left(x^{2}\right)}{1-\left(M_{\mathrm{VCA}}-M_{\alpha}-M_{\mathrm{VCA}} \Sigma\right) \omega^{2} F\left(\omega^{2}\right)}\right],
$$

where $x^{2}$ and $h\left(x^{2}\right)$ were defined below Eq. (22).

Within the CPA, the component densities of states have the pleasing property

$$
p g_{\mathrm{CPA}}^{(1)}(\omega)+(1-p) g_{\mathrm{CPA}}^{(2)}(\omega)=g_{\mathrm{CPA}}(\omega) .
$$

Thus, the total phase phonon density of states equals the sum of the properly weighted partial densities of states on the two species of grains. In our calculations, we have confirmed that this property is, indeed, satisfied for our particular choice of the Debye model.

\section{Spectral Function}

Our self-consistent set of equations permits calculation of another useful quantity: the spectral function, defined by

$$
\operatorname{Im} \bar{G}\left(\mathbf{q}, \phi^{2}\right)=\frac{\omega^{2} \operatorname{Im}\left[\Sigma\left(\omega^{2}\right)\right]}{\left\{\omega^{2}\left(1-\operatorname{Re}\left[\Sigma\left(\omega^{2}\right)\right]\right)-\omega_{\mathbf{q}}^{2}\right\}^{2}+\left\{\omega^{2} \operatorname{Im}\left[\Sigma\left(\omega^{2}\right)\right]\right\}^{2}} .
$$

The spectral function gives the frequency distribution of excitations of wave number $\mathbf{q}$ in the array, and, for our Debye model, depends only on the magnitude $q$. The full width at half maximum (FWHM) of the spectral function is inversely proportional to the decay time of the spinwave-like excitations at wave vector $\mathbf{q}$.

\section{Mean-Square Phase Fluctuations}

Finally, we can use our CPA calculations to infer the mean-square phase fluctuations at the $j$ th grain. We denote this quantity $\left\langle\left|\theta_{j}(t)\right|^{2}\right\rangle$, where $\langle\ldots\rangle$ denotes a quantum-mechanical average, and $\theta_{j}(t)$ denotes the phase of the $j$ th grain at time $t$. A quantum-mechanical average is appropriate at temperature $T=0$. At finite temperatures, one should carry out both a quantum and a thermal average. However, such an average diverges at $T \neq 0$ in two dimensions $(d=2)$, because of contributions from the long-wavelength phase phonons. In the present discussion, therefore, we discuss only the $T=0$ limit.

We will consider the quantity $\left\langle\left|\theta_{j}(t)\right|^{2}\right\rangle$ for a lattice with Josephson coupling $J$ and self-capacitance $C_{j}$, within the harmonic approximation. In this case, one can simply adapt the discussion of Ref. 15 for lattice vibrations in mass-disordered systems. In fact, the present problem is slightly easier since there are no polarization degrees of freedom. To obtain this quantity, we first use a result from Ref. 15 that

$$
\left\langle\theta_{j}(t) \theta_{k}(0)\right\rangle=-\frac{\hbar}{\pi} \operatorname{Im} \int_{-\infty}^{\infty} e^{i \omega t} G\left(j, k ; \varnothing^{2}\right) d \varnothing,
$$

where, at $T=0, G\left(j, k ; \varnothing^{2}\right)=\left\langle j\left|\frac{1}{\omega^{2}-H}\right| k\right\rangle$. The desired quantity is actually $\lim _{t \rightarrow 0}\left\langle\theta_{j}(t) \theta_{j}(0)\right\rangle$ averaged over disorder realizations, which we write

$$
\left\langle\left|\theta_{j}\right|^{2}\right\rangle_{\mathrm{dis}}=-\frac{\hbar}{\pi} \operatorname{Im} \int_{0}^{\infty} F\left(\varnothing^{2}\right) d \varnothing
$$

where we have used the definition of $F\left(z^{2}\right)$ in Eq. (6). The operators $\theta_{j}$ are computed at times $t=0$ (though this average is time-independent).

We first consider the virtual crystal lattice, in which $C_{j}=C_{\mathrm{VCA}}$ for all $j$. In this case, $\left\langle\left|\theta_{j}\right|^{2}\right\rangle$ is readily calculated from Eq. (28), using $F_{\mathrm{VCA}}(z)$ from Eq. (20), with the result

$$
\left\langle\left|\theta_{j}\right|^{2}\right\rangle_{\mathrm{VCA}}=\frac{\hbar}{\omega_{D} M_{\mathrm{VCA}}}
$$

where $\emptyset_{D}=\sqrt{4 \pi J / M_{\mathrm{VCA}}}=\sqrt{16 \pi e^{2} J /\left(\hbar^{2} C_{\mathrm{VCA}}\right)}$.

For the actual disordered lattice, we can calculate within the CPA not only the full disorder average of $\left\langle\left|\theta_{j}\right|^{2}\right\rangle_{\text {dis }}$, but also the disorder average of $\left\langle\left|\theta_{j}\right|^{2}\right\rangle$ over sites of type $\alpha$, which we denote $\left\langle\left|\theta_{j}\right|^{2}\right\rangle^{(\alpha)}$. The result is

$$
\begin{aligned}
\left\langle\left|\theta_{j}\right|^{2}\right\rangle^{(\alpha)} & =\frac{-\hbar}{\pi} \operatorname{Im} \int_{0}^{\infty} \bar{G}^{\alpha}\left(j, j ; \phi^{2}\right) d \varnothing \\
& =\frac{-\hbar}{\pi} \operatorname{Im} \int_{0}^{\infty} \frac{F\left(x^{2}\right)}{1-\left(M_{\mathrm{VCA}}-M_{\alpha}-M_{\mathrm{VCA}} \Sigma\right) \varnothing^{2} F\left(x^{2}\right)} d \varnothing
\end{aligned}
$$


(a) $\mathrm{p}=0.25$
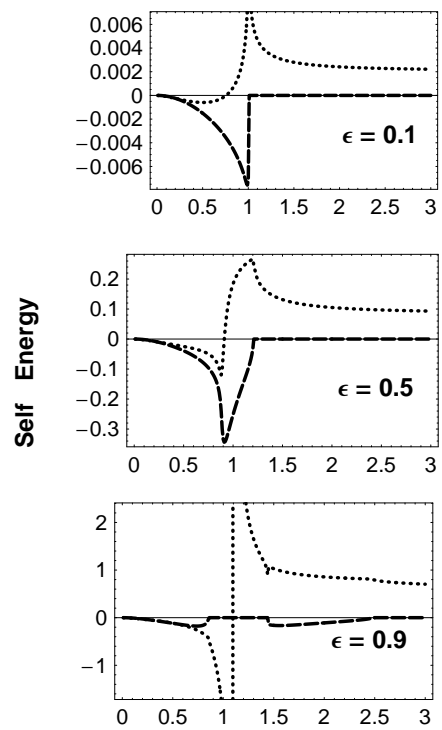

(b) $\mathrm{p}=0.75$
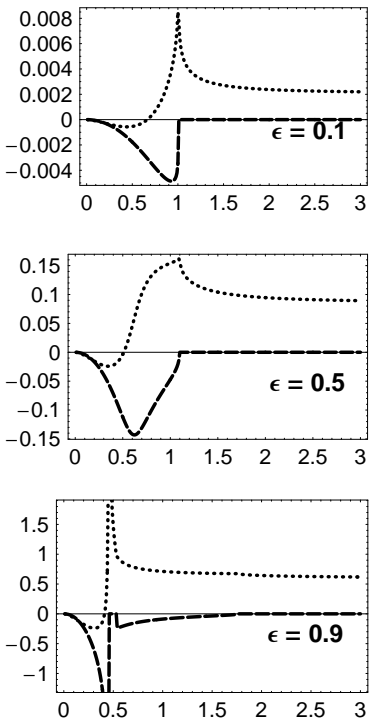

Frequency $\omega / \omega_{\mathrm{D}}$

FIG. 1: (a) The real (dotted line) and imaginary (dashed line) parts of the self-energy, $\Sigma(\varnothing)$, plotted as a function of the scaled frequency $\varnothing / \varnothing_{D}$ for three different cases: $\epsilon=0.1,0.5$, and 0.9 , at a concentration $p=0.25$ of light defects. Note that $\omega_{D}$ is a function of $p$ and $\epsilon$ [cf. Eq. (19p]. (b) Same as (a) except that $p=0.75$. Note the different vertical scales on different portions of the Figure.

where $x^{2}=\left(\omega^{2} / \omega_{D}^{2}\right)[1-\Sigma(\omega)]$. The full $\left\langle\left|\theta_{j}\right|^{2}\right\rangle_{\text {dis }}(p)$ in the CPA satisfies

$$
\left\langle\left|\theta_{j}\right|^{2}\right\rangle_{\operatorname{dis}}(p)=\sum_{\alpha} p_{\alpha}\left\langle\left|\theta_{j}\right|^{2}\right\rangle^{(\alpha)},
$$

where the sum runs over the two species $\alpha$, and in our notation $p_{1}=p$, and $p_{2}=1-p$.

We can use these equations to compute various ratios, such as $\left\langle\left|\theta_{j}\right|^{2}\right\rangle_{\text {dis }}(p) /\left\langle\left|\theta_{j}\right|^{2}\right\rangle_{\mathrm{VCA}}$, and hence to see how the mean-square phase fluctuations increase with increasing disorder.

\section{NUMERICAL RESULTS}

We have solved the model described in the preceding section to obtain the self-energy, density of states, spectral function, and mean-square phase fluctuations of a two-dimensional lattice, using a Debye phase phonon spectrum. The numerical solution are obtained from straightforward iterative solution of the three selfconsistent equations (12), (17), and (20). It is important to take care that the resulting solutions are the proper physical ones, with $\operatorname{Im} \Sigma(\omega) \leq 0$ and with both $F\left(z^{2}\right)$ and $\Sigma(z)$ varying smoothly with $z$.

(a) $\mathrm{p}=0.25$

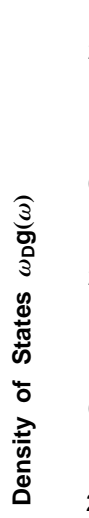

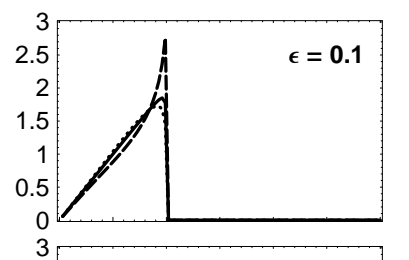
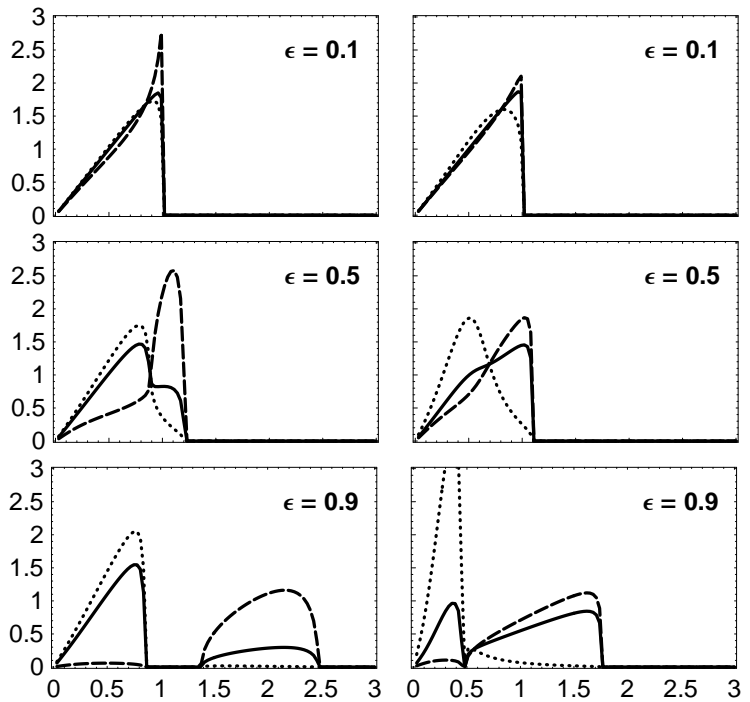

Frequency $\omega / \omega_{\mathrm{D}}$

FIG. 2: (a) Normalized density of states of the phase phonon excitations, plotted as a function of the scaled frequency $\varnothing / \varnothing_{D}$ calculated for a concentration $p=0.25$ of low-capacitance defects and three different values of the disorder parameter $\epsilon=1-C_{1} / C_{2}$, as indicated in the Figure. The total density of states for phase phonon excitations, $g_{\mathrm{CPA}}(\varnothing)$, is the solid line, while the component density of states $g_{\mathrm{CPA}}^{(1)}(\varnothing)$ and $g_{\mathrm{CPA}}^{(2)}(\varnothing)$ associated with low-capacitance and high-capacitance grains are the dashed and dotted lines, respectively. The concentration of light defects (type 1 ) is $p=0.25$. (b) same as (a) except that $p=0.75$. As in Fig. $1, \omega_{D}$ is a function of both $p$ and $\epsilon$ [Eq. (19)].

In Fig. 1 we show the real and imaginary parts of the self-energy, $\Sigma(\varnothing)$ (dotted and dashed curves respectively), plotted against the scaled frequency $\varnothing / \varnothing_{D}$, where $\emptyset_{D}=\left(4 \pi J / M_{\mathrm{VCA}}\right)^{1 / 2}$ is the virtual crystal Debye frequency. We have carried out our calculations for two concentrations of the low-mass defects (type 1 ), $p=0.25$ and $p=0.75$; the results are shown in Figs. 1(a) and 1(b). The three different panels correspond to different values of the ratio $\epsilon \equiv 1-C_{1} / C_{2}=0.1,0.5$, and 0.9 . These ratios range from small deviations in capacitance between type 1 and type $2(\epsilon=0.1)$ to large deviations $(\epsilon=0.9)$. Note the differences in the scales of the plots.

Given the self-energy, several other physical quantities can be calculated, as explained in Sec. III. For example, the averaged density of states of phase-phonon excitations can be obtained within the CPA from Eq. (22), while the averaged component densities of states follow from Eq. (24). In Fig. 2 we show the average density of states $g_{\mathrm{CPA}}(\omega)$ obtained within the CPA (solid line), as well as the component densities of states $g_{\mathrm{CPA}}^{(1)}(\varnothing)$ (dashed 

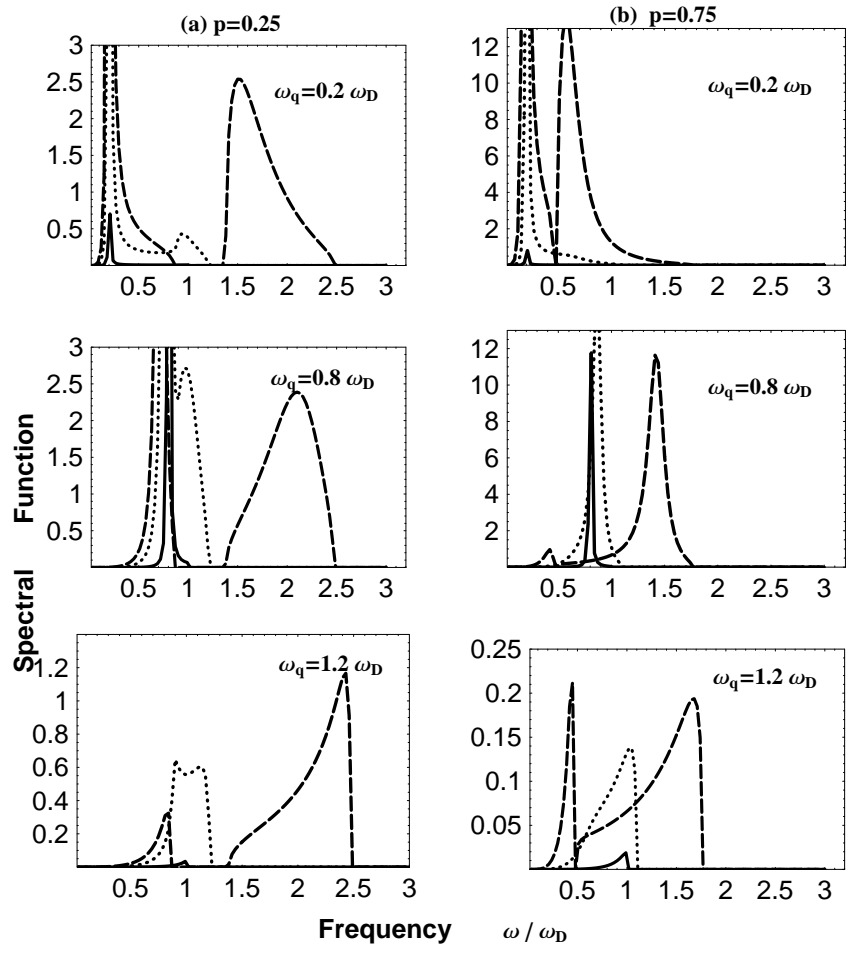

FIG. 3: (a) Spectral function $\operatorname{I} m \bar{G}\left(\mathbf{q}, \varnothing^{2}\right)$, plotted against the scaled frequency $\varnothing / \varnothing_{D}$ for three different mode frequencies $\omega_{\mathbf{q}}$ as indicated in each panel. The concentration of light defects is $p=0.25$. For each $\omega_{\mathbf{q}}$ we have considered $\epsilon \equiv$ $1-C_{1} / C_{2}=0.1$ (solid line), 0.5 (dotted line), and 0.9 (dashed line), corresponding to increasing amounts of disorder. For $\phi_{\mathbf{q}} / \phi_{D}<1$, the spectral function always exhibits a single sharp peaked centered at $\varnothing=\phi_{\mathbf{q}}$. (b) same as (a) except that $p=0.75$.

line) and $g_{\mathrm{CPA}}^{(2)}(\varnothing)$ (dotted line). The sum rule given in Eq. (25) is evidently satisfied by these three densities of states.

These results show characteristic features expected from CPA calculations. For the case where the capacitance of type 2 is close to that of type $1(\epsilon=0.1)$, the full and the two component densities of states are all very similar, and all resemble the virtual-crystal result. As $\epsilon$ increases, there start to be more conspicuous differences between the partial densities of states on the light-mass (type 1) and heavy-mass (type 2) grains. In particular, there is a clearly-developed band gap between the two classes of states for $\epsilon=0.9$. This behavior is well known as the "split-band" regime in the phonon problem. The light-mass phase phonons presumably correspond to localized modes, though this localization is not probed in the CPA.

In Fig. 3, we plot the spectral function defined in Eq. (26) as a function of the scaled frequency $\varnothing / \varnothing_{D}$ for several choices of parameters. Our calculations are carried out

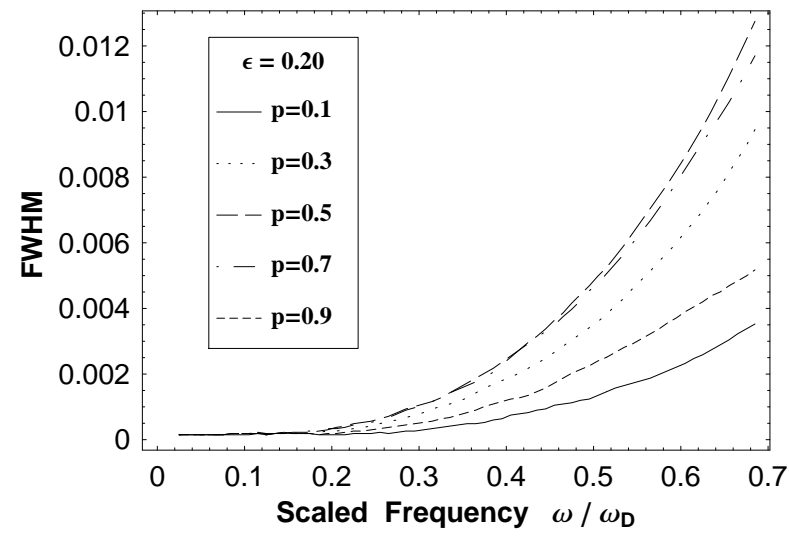

FIG. 4: The full width at half maximum (FWHM) of the spectral function, plotted versus scaled frequency $\varnothing / \emptyset_{D}$ for $\epsilon=$ 0.2 , and for several concentrations $p$ of the small-capacitance grains, as indicated in the legend.

at three different mode frequencies, $\varnothing_{\mathbf{q}} / \emptyset_{D}=0.2,0.8$, and 1.2, which are shown in the three different panels, and for two different defect concentrations, $p=0.25$ and $p=0.75$, which are shown in Figs. 3(a) and 3(b). In each of these cases, we have also considered three values of the mass ratio parameter: $\epsilon=0.1,0.5$, and 0.9 . (Note in particular the vertical scale changes in the Figures.) For $\emptyset_{\mathbf{q}} / \varnothing_{D}<1$, the spectral functions in all cases exhibit single sharp delta-function-like peaks centered at $\varnothing=\emptyset_{\mathbf{q}}$. However, for $\emptyset_{\mathbf{q}} / \emptyset_{D}>1$, there is some spectral strength which shows up as weak peaks at higher frequencies; these are presumably due to the localized modes in the light-mass bands. Note also that for $\epsilon=0.1$ there is almost no spectral weight for $\emptyset_{\mathbf{q}} / \phi_{D}>1$.

In each of the cases of Fig. 3, we have also calculated the full width of the spectral function peak at halfmaximum (FWHM). This width is inversely proportional to the lifetime of the phase phonon like excitations. In Fig. 4, we plot this FWHM as a function of $\varnothing_{\mathbf{q}} / \phi_{D}$ for $\epsilon=0.2$, and for several concentrations of the light defects as indicated in the legend. Evidently, for a given $\omega_{\mathbf{q}}$, the excitation lifetimes decrease, as expected, as $p$ increases from 0 to 0.5 , then increases again as $p$ increases from 0.5 to 1 . This behavior is consistent with the well-known result of second-order perturbation theory that this lifetime should vary approximately as $1 /[p(1-p)]$ in the weak-disorder regime. 8

Finally, we turn to the transition from phase coherence to phase incoherence in this system. If the model Hamiltonian is assumed to represent an array of small Josephson junctions, this corresponds to a superconductorinsulator (S/I) transition. It is believed that superconductivity in Josephson junction arrays is destroyed when the ratio of the charging energy to the Josephson cou- 

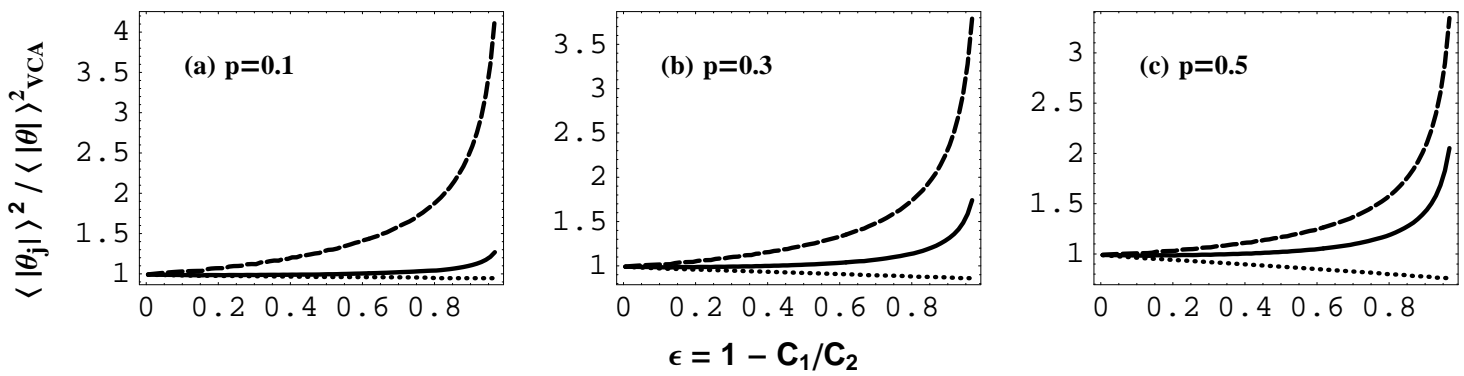

FIG. 5: (a). Mean-square phase fluctuation $\left\langle\left|\theta_{j}\right|^{2}\right\rangle_{\mathrm{dis}} /\left\langle\left|\theta_{j}\right|^{2}\right\rangle_{\mathrm{VCA}}$, as evaluated in the CPA, plotted as a function of $\epsilon=1-C_{1} / C_{2}$ for $p=0.1$. The solid line shows the CPA average value, the dashed line shows the mean-square phase fluctuations on a type 1 grain, and the dotted line on a type 2 grain. (b). same as (a) except that $p=0.3$. (c). same as (a) except that $p=0.5$.

pling energy exceeds some characteristic limiting value.l In fact, the S/I transition is a standard example of a $T=0$ quantum phase transition. In two-dimensional square arrays with nearest-neighbor coupling, it has been found experimentally that the S/I transition occurs near $U / J=1.7 \equiv(U / J)_{\mathrm{cr}}$.

In our discussion, we will assume a simple Lindemann melting criterion, namely, that superconductivity is destroyed when the mean fluctuations in the phase, given in Eq. (28), exceeds some limit to be determined below. (In $d=2$, such a criterion can be plausible only at $T=0$, since the mean-square phase fluctuation diverges at any finite $T$.) If all the grains have the same charging energy, then the mean-square fluctuation is given by Eq. (29), which implies a critical value of

$$
\left\langle\left|\theta_{j}\right|^{2}\right\rangle_{\mathrm{cr}}=(U / J)_{\mathrm{cr}}^{1 / 2} \approx 1.3
$$

Here $j$ can be any lattice site, and we have used $(U / J)_{\mathrm{cr}}=1.7$.

How can this Lindemann criterion be extended to a lattice with diagonal disorder in the charging energy? We speculate that a modified Lindemann criterion may still be usable, in the following way. Let us consider the model studied in this paper, in which there are two types of grains: "light" and "heavy" (or small and large capacitance). We suggest that a possible Lindemann criterion for destruction of superconductivity in this case is that the mean-square phase fluctuations, averaged over the two types of grains, should exceed the same critical value as in the ordered case.

In Fig. 5 we plot the calculated ratio $\left\langle\left|\theta_{i}\right|^{2}\right\rangle^{(\alpha)} /\left\langle\left|\theta_{i}\right|^{2}\right\rangle_{\mathrm{VCA}}$, which gives the mean-square phase fluctuations on grains of type $\alpha=1,2$ with respect to those of the virtual crystal (dashed and dotted lines). We also show $\left\langle\left|\theta_{i}\right|^{2}\right\rangle_{\text {dis }} /\left\langle\left|\theta_{i}\right|^{2}\right\rangle_{\mathrm{VCA}}$ which represents the average for the entire lattice (solid line). All quantities are calculated in the CPA as a function of $\epsilon=1-C_{1} / C_{2}$ for several different values of $p$, as indicated in the Figure. As expected, and as is clear from the Figure, for all values of $\left.p,\left\langle\left|\theta_{i}\right|^{2}\right\rangle^{(1)}\right\rangle\left\langle\left|\theta_{i}\right|^{2}\right\rangle^{(2)}$ because component 1 represents the light defects.

In principle, one might make an estimate of the critical value of $(U / J)^{1 / 2}$ at which melting occurs, as a function of $p$ and $\epsilon$, by assuming that melting occurs when $\left\langle\left|\theta_{i}\right|^{2}\right\rangle \geq 1.3$, as in the ordered lattice. Thus, given the properties of the lattice, one can easily extract an estimate of the melting parameters from this Figure.

\section{SUMMARY}

In this paper we have considered the behavior of a $2 \mathrm{D}$ quantum rotor model with diagonal disorder. In the case of Josephson junction arrays, this model corresponds to an array of small Josephson junctions with diagonal capacitive disorder. We have used the CPA to estimate the effects of disorder on the phase phonon density of states, the spectral functions, and the self-energy, all within the harmonic approximation to the original Hamiltonian. Finally, we obtained a crude estimate of the parameters governing the transition from coherence to incoherence in the disordered system, using a simple Lindemann criterion which may be applicable at $T=0$.

Viewed as a representation of a Josephson junction array (or a thin superconducting film), our model, and our approximations used to treat it, are both very simplified representations of a real superconducting array. For example, a bimodal distribution of charging energies is oversimplified, as is our assumption of diagonal charging energies and diagonal charging disorder. In addition, we have not considered dissipation, which is known to have important effects on S/I transitions in Josephson arrays. 11 Nevertheless, our results may provide some useful insights in understanding the S/I transition in such arrays, as well as the spectrum of excitations to be expected in disordered arrays.

Would it be possible to measure experimentally the phase phonon spectrum we have calculated in this paper? It is not clear what experiment would be directly 
sensitive to this spectrum in a superconducting array or film. A more promising direction might be the excitation spectrum of a Bose superfluid (e.g. ${ }^{4} \mathrm{He}$ ) in a porous medium. Such spectra have been extensively studied experimentally primarily using inelastic neutron scattering techniques. 16, 17, 18.19 While the behavior of ${ }^{4} \mathrm{He}$ in such porous media is certainly more complex than the relatively simple model discussed here, some aspects of the observed behavior (e. g., the persistence of rather sharp excitation peaks even in highly disordered systems) seems to be mirrored in our calculated spectral functions. Obviously, a more refined model, aimed specifically at the geometries of these porous glasses, is needed before any comparison to experiment can be contemplated.

Finally, we briefly comment on the quality of the approximation itself. While the CPA is an excellent mean- field approach, it is still based on a harmonic approximation to the underlying Hamiltonian. Ideally, it would be preferable to obtain the desired spectral functions using a more accurate approach, such as a quantum Monte Carlo technique (see, e.g., Ref. 20), or version of the self-consistent phonon approximation 1011 suitably generalized to treat a disordered system. We hope to return to such approaches in a future publication.

\section{ACKNOWLEDGMENTS}

This work has been supported by the National Science Foundation, through grant DMR01-04987.
* Electronic address: Al-Saidi.1@osu.edu

$\dagger$ Electronic address: Stroud@mps.ohio-state.edu

1 S. L. Sondhi, S. M. Girvin, J. P. Carini, and D. Shahar, Rev. Mod. Phys. 69, 315 (1997).

2 For a recent review see, for example, R. Fazio, and H. van der Zant, Phys. Rep. 355, 235 (2001).

3 For a recent review see, Y. Makhlin, G. Schön, and A. Shnirman, Rev. Mod. Phys. 73, 357 (2001) and references therein.

${ }^{4}$ C. R. Anderson, K. H. Andersen, W. G. Stirling, P. E. Sokol, and R. M. Dimeo, Phys. Rev. B 65, 174509 (2002).

5 P. Soven, Phys. Rev. 156, 809 (1967).

6 D. W. Taylor, Phys. Rev. 156, 1017 (1966).

7 R. J. Elliot and D. W. Taylor, Proc. Roy. Soc. (London) A 296, 161 (1967).

8 B. Velický, S. Kirkpatrick, and H. Ehrenreich, Phys. Rev. 175, 747 (1968).

${ }^{9}$ E. Simanek, Phys. Rev. B 22, 459 (1980).

10 D. M. Wood and D. Stroud, Phys. Rev. B 25, 1600 (1982).

11 S. Chakravarty, G.-L. Ingold, S. Kivelson, and A. Luther, Phys. Rev. Lett. 56, 2303 (1986), and references therein.

12 See, for example, M.-C. Cha, M. P. A. Fisher, S. M. Girvin,
M. Wallin, and A. P. Young, Phys. Rev. B 44, 6883 (1991).

13 K. C. Hass, M. A. Tamor, T. R. Anthony, and W. F. Banholzer, Phys. Rev. B 45, 7171 (1992).

14 In solving the CPA equations, one must always take care to choose the solutions which vary smoothly with frequency and which exhibit the proper analytical properties, for example $g_{\mathrm{CPA}}(\omega) \geq 0$.

15 R. J. Elliott, J. A. Krumhansl, and P. L. Leath, Rev. Mod. Phys. 46, 465 (1974).

16 J. De Kinder, G. Coddens, and R. Millet, Z. Phys. B: Condens. Matter 95, 511 (1994).

17 O. Plantevin, B. Fak, H. R. Glyde, J. Bossy, and J. R. Beamish, Phys. Rev. B 57, 10775 (1998).

18 C. R. Anderson, K. H. Andersen, J. Bossy, W. G. Stirling, R. M. Dimeo, P.E. Sokol, J. C. Cook, and D. W. Brown, Phys. Rev. B 59, 13588 (1999).

19 R. M. Dimeo, P. E. Sokol, C. R. Anderson, W. G. Stirling, K. H. Andersen, and M. A. Adams, Phys. Rev. Letters 81, 5860 (1998).

20 See, e. g., M. Wallin, E. S. Sorensen, S. M. Girvin, and A. P. Young, Phys. Rev. B 49, 12115 (1994). 